\title{
A 29-year-old Bodybuilder with Liothyronine-induced Thyrotoxic Hypokalaemic Periodic Paralysis
}

\author{
Querijn N E van Bokhorst ${ }^{1}$, Yvonne H M Krul-Poel ${ }^{1}$, Diederik L Smit ${ }^{2}$, Willem de Ronde ${ }^{3}$ \\ ${ }^{1}$ Department of Internal Medicine, Noordwest Ziekenhuisgroep, Alkmaar, The Netherlands \\ ${ }^{2}$ Department of Internal Medicine, Elisabeth-TweeSteden Ziekenhuis, Tilburg, The Netherlands \\ ${ }^{3}$ Department of Internal Medicine, Spaarne Gasthuis, Haarlem, The Netherlands
}

\begin{abstract}
Received: 05/02/2020
Accepted: 08/02/2021

Published: 04/03/2021 hypokalaemic periodic paralysis. EJCRIM 2021;8: doi:10.12890/2021_002362.

Conflicts of Interests: The Authors declare that there are no competing interests.

This article is licensed under a Commons Attribution Non-Commercial 4.0 License
\end{abstract}

How to cite this article: van Bokhorst QNE, Krul-Poel YHM, Smit DL, de Ronde W. A 29-year-old bodybuilder with liothyronine-induced thyrotoxic

\section{ABSTRACT}

We describe a 29-year-old male bodybuilder with recurrent attacks of myalgia and muscle weakness associated with hypokalaemia and thyrotoxicosis due to abuse of liothyronine. The attacks quickly resolved after potassium supplementation and liothyronine cessation. We concluded that the patient had thyrotoxic hypokalaemic periodic paralysis (TPP). Although muscle weakness and hypokalaemia are prominent symptoms of TPP, underlying thyrotoxicosis may be overlooked. Up to $25 \%$ of androgen abusers also abuse thyroid hormone. Lack of recognition of thyroid hormone abuse as a cause of hypokalaemic periodic paralysis may result in unnecessary, potentially harmful medical investigations and improper treatment and advice.

\section{LEARNING POINTS}

- In patients with bouts of muscle weakness and hypokalaemia, thyrotoxic hypokalaemic periodic paralysis should be suspected and thyroid function should be evaluated.

- In bodybuilders and strength athletes, undisclosed abuse of performance and image-enhancing drugs, including thyroid hormone, should be suspected.

\section{KEYWORDS}

Thyrotoxicosis, hypokalemic periodic paralysis, liothyronine, androgens

\section{CASE DESCRIPTION}

A 29-year-old Caucasian male presented at the emergency room with a 3-day history of progressive symptoms of myalgia and muscle weakness. Over a period of 6 years prior to presentation, he had experienced six similar episodes of acute muscle weakness, with spontaneous recovery within hours. At previous emergency room presentations and during previous hospital admissions, laboratory tests were unremarkable except for varying serum potassium levels with incidental hypokalaemia. Laboratory tests for the evaluation of thyroid function had not been performed previously. Extensive neurological evaluation, including spinal fluid analysis, magnetic resonant imaging of the spine and electromyography had not shown any abnormalities.

The patient was a heavyweight bodybuilder and admitted the use of performance-enhancing drugs, including testosterone enanthate, masteron enanthate, metandienone and human growth hormone. He also admitted the use of liothyronine on a regular basis at a dose of 
50-100 $\mu \mathrm{g}$ per day over the past 8 years. Physical examination at presentation showed a muscular young man (weight $128 \mathrm{~kg}$, height 190 $\mathrm{cm}$ ), with a blood pressure of $133 / 51 \mathrm{mmHg}$ and a pulse of $110 \mathrm{bpm}$. Neurological examination revealed mild muscle weakness in the legs and arms but was otherwise unremarkable. The patient did not report diarrhoea and denied the use of diuretics.

Blood tests were consistent with the use of exogenous liothyronine and showed significant hypokalaemia (Table 1). Oral potassium supplementation was started and myalgia and muscle weakness recovered spontaneously over the course of a few hours. The patient was explicitly advised to stop using liothyronine and was discharged without further treatment.

\begin{tabular}{|l|c|c|c|}
\hline & First Presentation & After 2 weeks & After 3 weeks \\
\hline $\begin{array}{l}\text { Potassium } \\
(3.4-4.9 \mathrm{mmol} / \mathrm{l})\end{array}$ & 2.7 & 1.5 & 4.3 \\
\hline $\begin{array}{l}\text { FT3 } \\
(3.5-6.5 \mathrm{pmol} / \mathrm{l})\end{array}$ & 15.4 & 2.1 & 4.5 \\
\hline $\begin{array}{l}\text { FT4 } \\
(11.0-21.0 \mathrm{pmol} / \mathrm{l})\end{array}$ & 3.3 & 3.1 & 11.2 \\
\hline $\begin{array}{l}\text { TSH } \\
(0.35-5.0 \mathrm{mU} / \mathrm{l})\end{array}$ & 0.01 & 0.01 & 2.8 \\
\hline
\end{tabular}

Table 1. Laboratory results for potassium and thyroid function at first presentation and follow-up

Despite initial improvement, symptoms of muscle weakness persisted over subsequent weeks. Two weeks after the initial presentation, the patient again presented to the emergency unit because of rapid progression of muscle weakness accompanied by myalgia and heavy chest pain. Blood tests revealed severe hypokalaemia, suppressed concentrations of TSH and free T4, but a normal level of free T3 (Table 1). Despite strict medical advice, the patient admitted to having used T3 since the last presentation. Neurological examination provided no new symptoms in comparison to previous episodes.

The patient was admitted for intravenous potassium supplementation. Other causes for hypokalaemia (i.e. dietary deficiencies, diarrhoea, medication, alkalosis) were ruled out. With normalization of serum potassium levels, the muscle weakness resolved. From this point on, the patient completely abstained from exogenous T3. Blood tests for thyroid function were repeated 1 week later, and showed normal thyroid function. Genetic screening did not show any mutations in the CACNA1S and SCN4A genes.

\section{DISCUSSION}

We present a patient with bouts of myalgia and muscle weakness associated with hypokalaemia and misuse of thyroid hormone. After exclusion of other, more common causes of hypokalaemia and muscle weakness, we concluded that the patient had thyrotoxic periodic paralysis (TPP). This is a rare, but serious complication of thyrotoxicosis, characterized by episodes of muscle paralysis and hypokalaemia. An attack is characterized by recurrent, transient episodes of muscle weakness that range from mild weakness to complete paralysis. Patients may experience recurrent episodes of weakness that last from a few hours to up to 72 hours, with complete recovery between the attacks. There may also be prodromal symptoms of aches, cramps and stiffness of the affected muscles ${ }^{[1,2]}$.

The symptoms are the result of a massive shift of potassium from the extracellular compartment into the intracellular compartment. This shift is facilitated by an increased number and activity of sodium-potassium pumps in the cell membrane of thyrotoxic patients. Attacks may be triggered by circumstances that induce intracellular influx of potassium, such as hyperinsulinaemia, adrenergic activity and recovery after exercise ${ }^{[1,2]}$.

In Western countries, the incidence of this syndrome is low, probably being around $0.1-0.2 \%$ in thyrotoxic patients. Patients of Asian descent are much more affected than Caucasians, which suggests a genetic predisposition to the development of TPP. Mutations in the CACNA1S and SCN4A genes that are associated with familial hypokalaemic periodic paralysis are normally absent ${ }^{[2]}$. Recently it was demonstrated that up to $33 \%$ of patients with TPP have a mutation in the gene that encodes for Kir 2.6, a potassium channel in muscle cells that is regulated by thyroid hormone ${ }^{[3]}$. Males are much more affected than females. The reason for this is unknown but suggests androgens have a role in the pathogenesis of TPP. The condition is normally seen in patients with thyroid dysfunction such as Graves' disease and toxic goitre, which are the most common causes of hyperthyroidism. However, TPP has been described in patients with thyrotoxicosis with all possible causes, including thyroid hormone abuse ${ }^{[1,2]}$ 
The abuse of thyroid hormone is well established among androgen abusers for the purpose of reducing fat mass. In a recent survey in our clinic, $15 \%$ of androgen abusers also included thyroid hormone in their anabolic steroid regime ${ }^{[4]}$ and $23 \%$ reported they had ever used thyroid hormone for non-medical reasons [5]. Adrenergic drugs, such as clenbuterol, ephedrine and caffeine, are also used by a considerable number of androgen abusers to reduce fat mass or to improve training intensity ${ }^{[4,5]}$. In combination with strenuous exercise, all these factors may precipitate TPP.

In the acute care setting, rapid reversal of hypokalaemia is the first step in treating TPP. However, since there is no actual body potassium deficit, massive potassium supplementation is contraindicated to prevent rebound hyperkalaemia. Oral or intravenous administration of propranolol has been advocated to rapidly reverse muscle weakness. Obviously, the cause of thyrotoxicosis should be established and treated. In the meantime, the patient should be instructed to avoid strenuous exercise and high carbohydrate diets. Oral propranolol may be effective to prevent attacks while thyrotoxicosis is being resolved ${ }^{[2,3]}$.

In conclusion, this case shows that in patients with recurrent episodes of muscle weakness and hypokalaemia, TPP should be included in the differential diagnosis and thyroid function should be evaluated. Additionally, in young, muscular men, undisclosed use of thyroid hormone should be suspected.

\section{REFERENCES}

1. Kung A. Thyrotoxic periodic paralysis: a diagnostic challenge. J Clin Endocrinol Metab 2006;91(7):2490-2495.

2. Maciel R, Lindsey S, Dias da Silva M. Novel etiopathophysiological aspects of thyrotoxic periodic paralysis. Nat Rev Endocrinol 2011;7(11):657-667.

3. Ryan D, Dias da Silva M, Soong T, Fontaine B, Donaldson M, Kung A et al. Mutations in potassium channel Kir2.6 cause susceptibility to thyrotoxic hypokalemic periodic paralysis. Cell 2010;140(1):88-98.

4. Smit D, Hon O, Venhuis B, den Heijer M, Ronde W. Baseline characteristics of the HAARLEM study: 100 male amateur athletes using anabolic androgenic steroids. Scand J Med Sci Sports 2019;30(3):531-539.

5. Smit D, de Ronde W. Outpatient clinic for users of anabolic androgenic steroids: an overview. Neth J Med 2018;76(4):167. 